\title{
RELATION BETWEEN HIGHER OBSTRUCTIONS AND POSTNIKOV INVARIANTS
}

\author{
KENICHI SHIRAIWA*
}

\section{Introduction}

The problem of extending a continuous map is one of the most important problems in algebraic topology. Many topologists have contributed for the solution of this problem. One of the most powerful methods in the extension problem is the obstruction theory defined first by S. Eilenberg [1] and developed by many others. N. E. Steenrod worked on the primary obstruction and showed that there is a strong connection between obstruction theory and cohomology operations [7].

The main objective of this paper is to establish a certain relation between higher obstructions and the higher order cohomology operations induced by the Postnikov invariants in the sense of F. P. Peterson [4]. But this is done only for the stable range.

\section{Obstruction set}

By a pair $(K, L)$ we shall mean a connected CW-complex $K$ together with a subcomplex $L \subset K$. Let $K^{q}$ be a $q$-skeleton of $K$ and $\bar{K}^{q}=K^{q \cup} L$. Let $f: \bar{K}^{q}$ $\rightarrow X(q \geqq 1)$ be a map from $\bar{K}^{q}$ to a topological space $X$, which is $n$-simple for all $n \geqq 1$. Then we define the obstruction cocycle $c^{q+1}(f) \in Z^{q+1}\left(K, L ; \pi_{q}(X)\right)$ in a usual fashion, where $Z^{q+1}\left(K, L ; \pi_{q}(X)\right)$ is the $(q+1)$-dimensional group of cocycles of $K$ modulo $L$ with coefficients in $\pi_{q}(X)$ and $\pi_{q}(X)$ is the $q$-dimensional homotopy group of the space $X$.

Let $f_{0}$ and $f_{1}$ be maps of $\bar{K}^{q}$ to $X$ which agree on $\bar{K}^{q-1}$, then we can define a difference cochain $d^{q}\left(f_{0}, f_{1}\right) \in C^{q}\left(K, L ; \pi_{q}(X)\right)$ in a usual fashion, where $C^{q}\left(K, L ; \pi_{q}(X)\right)$ is the $q$-dimensional cochain group of $K$ modulo $L$ with coefficients in $\pi_{q}(X)$. Then the following properties are well known. (Cf. Eilenberg

Received June 25, 1959.

* The author was partly supported by the office of Ordnance Research, U.S. Army (contract DA-30-069-ORD-2194) during the preparation of this paper. 


\section{[1], Olum [3], Steenrod [7])}

(1.1) If $f_{0} \sim f_{1}$ (i.e. $f_{0}$ and $f_{1}$ are homotopic), then $c^{q+1}\left(f_{0}\right)=c^{q+1}\left(f_{1}\right)$.

(1.2) $c^{q+1}(f)=0$ if and only if $f$ extends over $\bar{K}^{q+1}$.

(1.3) $\delta d^{q}\left(f_{0}, f_{1}\right)=c^{q+1}\left(f_{0}\right)-c^{q+1}\left(f_{1}\right)$, where $\delta$ is the coboundary operation.

(1.4) If $f_{0}$ and $d \in C^{q}\left(K, L ; \pi^{q}(X)\right)$ are given, there exists a map $f_{1}$ such that $d^{q}\left(f_{0}, f_{1}\right)=d$.

(1.5) $d^{q}\left(f_{0}, f_{1}\right)+d^{q}\left(f_{1}, f_{2}\right)=d^{q}\left(f_{0}, f_{2}\right)$.

(1.6) $d^{q}\left(f_{0}, f_{1}\right)=0$ if and only if $f_{0} \sim f_{1}$ relative to $\bar{K}^{q-1}$.

(1.7) If $g:(K, L) \rightarrow\left(K^{\prime}, L^{\prime}\right)$ is cellular and $f: \bar{K}^{\prime q} \rightarrow X$, then $g^{\sharp} c^{q+1}(f)$ $=c^{q++1}(f \circ g)$,

where $g^{\#}$ is an induced cochain map of $g$.

Let $f: \bar{K}^{q} \rightarrow X$, we define $O_{q}^{r}(f)$ for $r>q+1$ as a set of all obstruction cocycles $c^{r}\left(f^{\prime}\right)$, where $f^{\prime}$ is an extension of $f$ over $\bar{K}^{r-1}$. Then $O_{q}^{q+2}(f)$ is either an empty set or a single cohomology class by (1.3) and (1.4). Therefore, we denote by $\bar{c}^{q+2}(f)$ the cohomology class $O_{q}^{q+2}(f)$ if $f$ is extendable over $\bar{K}^{q+1}$. Next we define $Z_{q}^{r}(f)$ for $r>q+1$ as a set of all $\bar{c}^{-r}\left(f^{\prime}\right)$ of an extension $f^{\prime}: \bar{K}^{r-2} \rightarrow X$ of $f$ which is extendable over $K^{r-1}$. Then we have the following equality.

(1.8) $O_{q}^{r}(f)=$ the set of all cocycles whose cohomology classes belong to $Z_{q}^{r}(f)$. Therefore, by (1.2) we have

(1.9) $Z_{q}^{r}(f) \ni 0$-cohomology class if and only if $f$ is extendable over $\bar{K}^{r}$.

\section{Postnikor system}

Let $X^{\prime}$ be a simply connected CW-complex, then there exists a sequence of fiber spaces $p_{n+1}: X_{n+1} \rightarrow X_{n}(n \geqq 2)$ such that

(2.1) $X^{\prime}$ and $X$ are of the same homotopy type, where $X$ is the inverse limit of $X_{n}$,

(2.2) the fiber of $p_{n+1}: X_{n+1} \rightarrow X_{n}$ is a $\left(\pi_{n+1}\left(X^{\prime}\right), n+1\right)$-type complex, (i.e. homotopy groups of the fiber vanish except for the $(n+1)$-st homotopy group which is isomorphic to $\left.\pi_{n+1}\left(X^{\prime}\right)\right)$

(2.3) $\pi_{q}\left(\pi_{n}\right)=0$ for $q>n$,

$$
p_{n+1 *}: \pi_{q}\left(X_{n+1}\right) \approx \pi_{q}\left(X_{n}\right) \text { for } q \leqq n \text {, and }
$$

(2.4) $p_{n+1}$ is an homeomorphism on the $n$-skeleton of $X_{n+1}$, and its inverse over the $n$-skeleton of $X_{n}$ is extendable over the $(n+1)$-skeleton of $X_{n}$ as a cross-section. 
The sequence of fiber spaces $\left\{\left(X_{n+1}, X_{n}, p_{n+1}\right)\right\}$ is by definition the Postnikov system in the sense of Moore. (Cf. Moore [2].)

By (2.2) and the classification theorem for fiber spaces with fiber a $(\pi, n)$. type space, we obtain a map $\left.k^{n-2}: X_{n} \rightarrow K\left(\pi n+1, X^{\prime}\right), n+2\right)$ such that the fiber space $\left(X_{n+1}, X_{n}, p_{n+1}\right)$ is equivalent to the induced fiber space of the standard fiber space over $K\left(\pi_{n+1}\left(X^{\prime}\right), n+2\right)$ by $k^{n+2}$, where the standard fiber space over the Eilenberg-MacLane complex $K\left(\pi_{n+1}\left(X^{\prime}\right), n+2\right)$ is the fiber space whose total space is contractible. To define the Postnikov invariants, we need a following well-known lemma.

LEMma 2.5.

$$
\pi\left(K, K\left(\pi^{\prime}, n\right)\right) \approx H^{n}\left(K, \pi^{\prime}\right),
$$

where $\pi\left(K, K\left(\pi^{\prime}, n\right)\right)$ is a group whose elements are homotopy classes of mappings $K \rightarrow K\left(\pi^{\prime}, n\right)$ with multiplication induced by the group structure of $K\left(\pi^{\prime}, n\right)$, and $H^{n}\left(K, \pi^{\prime}\right)$ is the $n$-th cohomology group of $K$ with coefficients in $\pi^{\prime}$.

By the above lemma we have a unique cohomology class $k^{n+2}(X)$ in $H^{n+2}\left(X_{n}, \pi n+1\left(X^{\prime}\right)\right)$ corresponding to $k^{n-2}: X_{n} \rightarrow K\left(\pi n+1\left(X^{\prime}\right), n+2\right) . \quad\left\{k^{n+2}\left(X^{n}\right)\right\}$ is by definition the Postnikov invariants of $X$, and it is known that $k^{n+2}(X)$ is equal to the primary obstruction of the fiber space $\left(X_{n+1}, X_{n}, p_{n+1}\right)$. (Cf. Moore [2].)

Let $f: K^{q} \rightarrow X^{\prime}$ be any map and $h: X^{\prime} \rightarrow X$ be an homotopy equivalence given in (2.1), then it is easy to see that the obstruction for extending $f$ is equivalent to that for $h \circ f$. Therefore, we can assume that $X^{\prime}=X$ for the rest of the paper.

Let $f: K^{q} \rightarrow X$ be extendable over $K^{q+1}$. Then $p_{q}^{\prime} \circ f: K^{q} \rightarrow X_{q}$ is extendable over $K$ since $X_{q}$ has vanishing homotopy groups $\pi i(X)$ for $i>q$, where $p_{q}^{\prime}: Y$ $\rightarrow X_{q}$ is a natural projection. Let $F: K \rightarrow X_{q}$ be any extension of $p_{y}^{\prime} \circ f$. Then

LEMMA 2.6.

$$
\bar{c}^{q+2}(f)=F^{*} k^{\eta+2}(X),
$$

where $F^{3}: H^{q+2}\left(X_{q}, \pi_{q+1}(X)\right) \rightarrow H^{q+2}\left(K, \pi_{q+1}(X)\right)$ is the induced homomorphism of $F$.

Proof. This is an easy consequence of the fact that $k^{q+2}(X)$ is also the primary obstruction for the fiber space $p_{q}^{\prime}: Y>X_{q}^{\prime}$, and it is equal to the obstruction for extending the cross-section as a continuous map for this fiber space. 
Suppose $f: K^{q} \rightarrow X$ be extendable over $K^{r+1}, r \geqq q$, then $p_{r}^{\prime} \circ f: K^{q} \rightarrow X_{r}$ is extendable over $K$ by the same argument above. By Lemma 2.6, we immediately have

Lemma 2.7. $Z_{q}^{r+2}(f)$ is the set of all $F^{*} k^{r+2}(X)$; where $F: K \rightarrow X_{r}$ is an extension of $p_{r}^{\prime} \circ f: K^{q} \rightarrow X_{r}$.

\section{Higher order cohomology operations}

We shall give a brief resume of higher order cohomology operations in the sense of Peterson (for details and proofs see Peterson [4]).

Let $\left\{\left(X_{q+1}, X_{q}, p_{q+1}\right)\right\}$ be a Postnikov system, and suppose $X_{n}=K\left(\pi_{n}, n\right)$. Consider the following diagram.

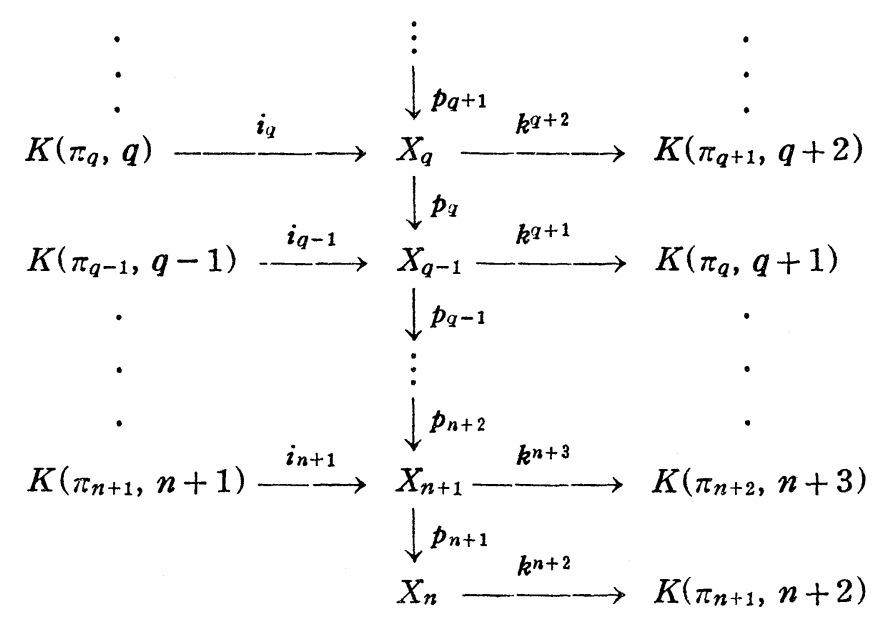

Diagram 1.

where $p_{q}: X_{q} \rightarrow X_{q-1}$ is the fiber map of the Postnikov system, $i_{q}: K\left(\pi_{q}, q\right) \rightarrow X_{q}$ is the inclusion map of the fiber into the total space of the fibering $X_{q} \rightarrow X_{q-1}$, $k^{q+2}: X_{q} \rightarrow K\left(\pi_{q+1}, q+2\right)$ is the map corresponding to the postnikov invariant of $X$, and $\pi_{q}=\pi_{q}(X)$.

Assume that each $k^{q+2}(X)$ is a suspension for the rest of this section. Applying the functor $X \rightarrow \pi(K, X)$ to the diagram 1, we have the diagram 2, by using Lemma 2.5 . 


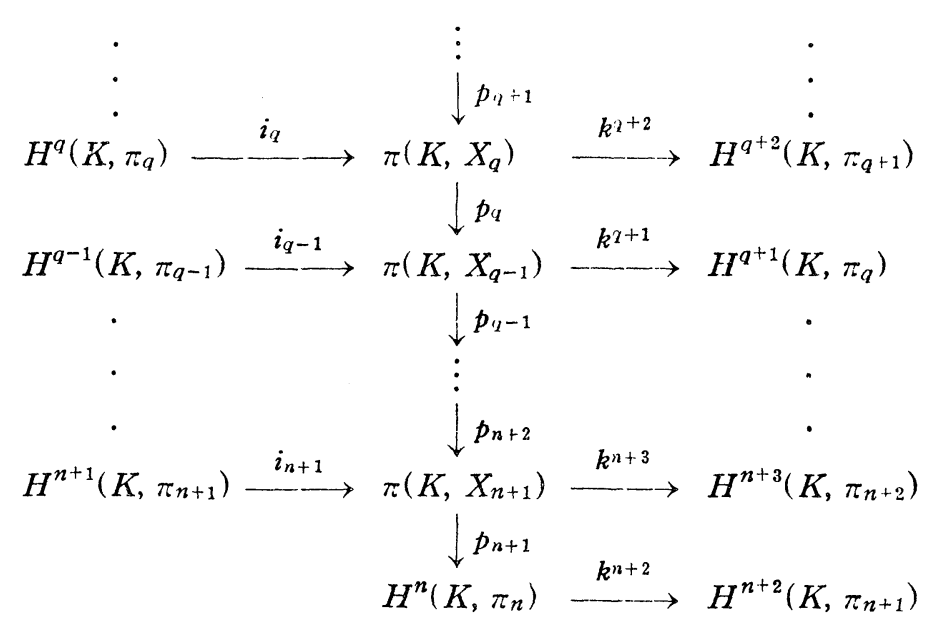

Diagram 2.

In the above diagram we have the following properties.

(3.1) Each object in the diagram 2 is a group and each map is a homomorphism.

(3.2) The sequence

$$
H^{q}\left(K, \pi_{q}\right) \stackrel{i_{q}}{\longrightarrow} \pi\left(K, X_{q}\right) \stackrel{p_{q}}{\longrightarrow} \pi\left(K, X_{q-1}\right) \stackrel{k^{2+1}}{\longrightarrow} H^{q^{+1}}\left(K, \pi_{q}\right)
$$

is exact.

(3.3) $H^{n}\left(K, \pi_{n}\right) \stackrel{k^{n+2}}{\longrightarrow} H^{n+2}\left(K, \pi_{n+1}\right)$ is the ordinary cohomology operation corresponding to $k^{n+2}(X) \in H^{n+2}\left(\pi_{n}, n, \pi_{n+1}\right)$, where $H^{n+2}\left(\pi_{n}, n, \pi_{n+1}\right)$ is the $(n+2)$-nd cohomology group of the Eilenberg-MacLane complex $K\left(\pi_{n}, n\right)$ with coefficients in $\pi_{n+1}$.

Let $x \in H^{n}\left(K, \pi_{n}\right)$ be an element such that $k^{n+2}(x)=0$. By (3.2) we have an element $y \in \pi\left(K, X_{n+1}\right)$ such that $p_{n+1}(y)=x$. Then $k^{n+3}(y)$ is unique modulo the image of $H^{n+1}\left(K, \pi_{n+1}\right)$ under the mapping $k^{n+3} \circ i_{n+1}$. We denote by $\left[k^{n+3}\right]$ the operation $x \rightarrow k^{n+3}(y)$ modulo Image $\left(k^{n+3} \circ i_{n+1}\right)$.

In the same fashion we define $\left[k^{q+2}\right]$ from the Kernel $\left(\left[k^{q+1}\right]\right)$ into $H^{q+2}\left(K, \pi_{q+1}\right)$ modulo a certain subgroup $L^{q+2}\left(k^{q+2}\right)$. Then we have the follow. ing lemma.

Lemma 3.4. If $x \in$ Kernel $\left(\left[k^{q+1}\right]\right)$, then $\left[k^{q+2}\right] x$ is the set of all $F^{*} k^{q+2}(X)$, where $F: K \rightarrow X_{q}$ is a map such that $p_{n+1} \circ \cdots \circ p_{q} \circ F=\bar{x}$, and $\bar{x}: K \rightarrow K\left(\pi_{n}, n\right)$ is a map corresponding to $x \in H^{n}\left(K, \pi_{n}\right)$. 
The proof of the lemma will follow immediately from the definition of $\left[k^{q \vdash 2}\right]$.

Lemma 3.5. $x \in$ Kernel $\left(\left[k^{q+1}\right]\right)$ if and only if there exists a map $G: K$ $\rightarrow X_{q}$ such that $p_{n+1} \circ \cdots \circ p_{q} \circ G=\bar{x}$ where $\bar{x}: K \rightarrow K\left(\pi_{n}, n\right)$ is a map corresponding to $x \in H^{n}\left(K, \pi_{n}\right)$.

Proof. By Lemma 3.4 we set $p_{q} \circ G=F$, then we immediately have $F^{*} k^{q+1}(X)=0$. The converse is also straight forward.

\section{Main theorems}

Let $X$ be $(n-1)$-connected $(n \geqq 2)$. Suppose that its Postnikov invariants $k^{q+2}(X)$ are suspensions for $q+2 \leqq r$. Then each $k^{q+2}(X)$ defines a higher order cohomology operation $\left[k^{q+2}(X)\right]$ for $q+2 \leqq r$. Let $b^{n}(X) \in H^{n}\left(X, \pi_{n}(X)\right)$ be the element corresponding to the inverse of the Hurewicz isomorphism, i.e. the fundamental cohomology class of $X$.

TheOREM 4.1. Let $X,\left[k^{q+2}(X)\right]$ be as above. Let $f: K^{n} \rightarrow X$ be extendable over $K^{q+1}$ for $n \leqq q \leqq r-2$. Then $Z_{n}^{q+2}(f)$ is a coset of $H^{q+2}\left(K, \pi_{q+1}(X)\right)$ by $L^{q+2}\left(k^{q+2}(X)\right)$, and $f^{*}\left(b^{n}(X)\right)$ is in the image of $i^{*}: H^{n}(K) \rightarrow H^{n}\left(K^{n}\right)$, where $i^{*}$ is an isonorphism into induced by the injection $i: K^{n} \rightarrow K$. Furthermore, $i^{*-1} \circ f^{*}\left(b^{n}(X)\right)$ is in the Kernel $\left(\left[k^{q ! 1}(X)\right]\right)$, and the following equality holds.

$$
Z_{n}^{q+2}(f)=\left[k^{q+2}(X)\right]\left\{i^{*-1} \circ f^{*}\left(b^{n}(X)\right)\right\} .
$$

Proof. Since $f$ is extendable over $K^{q+1}(q \geqq n), f^{*}\left(b^{n}(X)\right)$ is in the image of $i^{*}$, and by Lemma 3.5 we have $i^{*-1} \circ f^{*}\left(b^{n}(X)\right) \in$ Kernel $\left(\left[k^{q+1}(X)\right]\right)$. Therefore, by Lemma 3.4 we have $\left[k^{q+2}(X)\right]\left\{i^{*-1} \circ f^{*}\left(b^{n}(X)\right)\right\}$ is the set of all $F^{*} k^{q+2}(X)$, where $F: K \rightarrow X_{q}$ is a map such that $p_{n+1} \circ \cdots \circ p_{q} \circ F$ is an extension of $p_{n}^{\prime} \circ f$. It is obvious that $Z_{n}^{q+2}(f)$ coincides with $\left[k^{n+2}(X)\right]\left\{i^{*-1}\right.$ 。 $\left.f^{*}\left(b^{n}(X)\right)\right\}$ by Lemma 2.7 .

Remark. If $X$ is $(n-1)$-connected $(n \geqq 2)$, then $k^{q+2}(X)$ are suspensions for $q+2 \leqq 2 n-1$.

Corollaky 4.2. Let $X$ be $(n-1)$-connected $(n \geqq 3)$, and $f: K^{n} \rightarrow X$ be extendable over $K^{n\llcorner 1}$. Then

$$
\bar{c}^{n \div 2}(f)=S q^{2}\left\{i^{*-1} \circ f^{*}\left(b^{n}(X)\right)\right\} .
$$

Proof. Let $\phi: \pi n(X) \rightarrow \pi_{n}(X)$ be the pairing induced by composing the 
essential map of $S^{n-1}$ to $S^{n}$, then it induces the squaring operation $S q^{2}$ : $H^{n}\left(K, \pi_{n}(X)\right) \rightarrow H^{n+2}\left(K, \pi_{n+1}(X)\right)$. It is well-known that $k^{n+2}(X)=S q^{2}\left(b^{n}(X)\right)$.

(Cf. J. H. C. Whitehead [8].) Therefore, the corollary is proved.

For the next theorem we need the following diagarm.

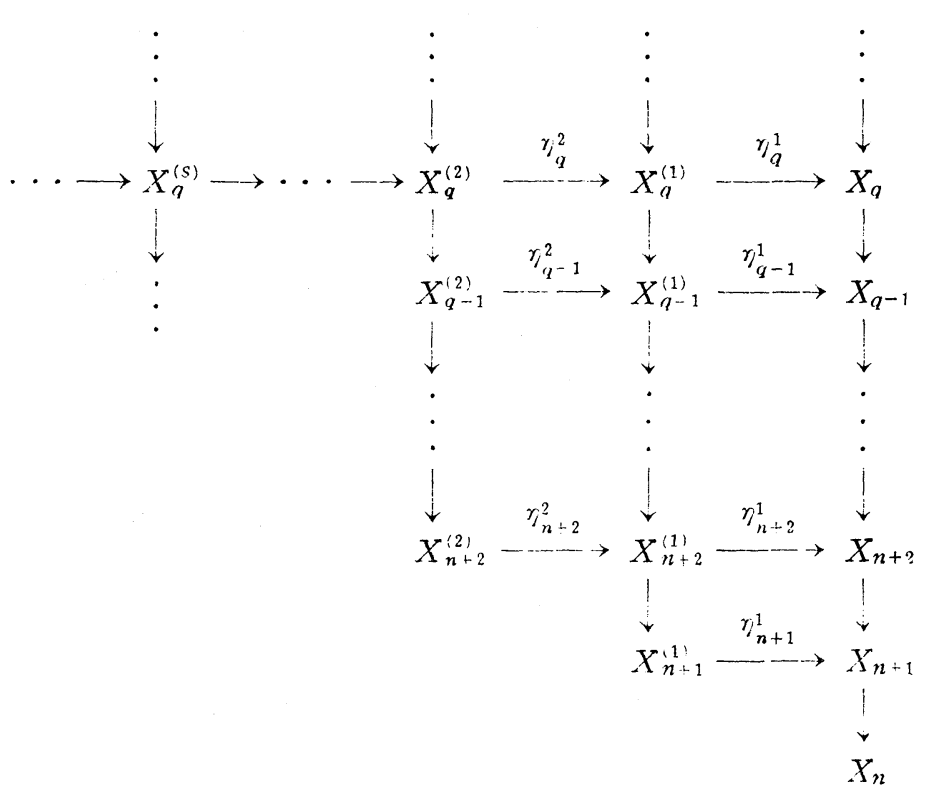

Diagram 3.

The above diagram is constructed as follows.

$\eta_{n+1}^{1}: X_{n+1}^{(1)} \rightarrow X_{n+1}$ is the injection of the fiber $X_{n+1}^{(1)}$ of the fiber space $p_{n+1}: X_{n+1} \rightarrow X_{n} . \quad X_{s}^{(1)} \rightarrow X_{s-1}^{(1)}(s \geqq n+2)$ is the induced fiber space of $X_{s} \rightarrow X_{s-1}$ by $\gamma_{s-1}^{1}: X_{s-1}^{(1)} \rightarrow X_{S-1}$, and $\eta_{s}^{1}: X_{S}^{(1)} \rightarrow X_{S}$ is the induced fiber space map. Then the sequence of fiber spaces $\left\{X_{S}^{(1)} \rightarrow X_{S-1}^{(1)}\right\}$ forms the Postnikov system of the space $X^{(1)}$ which is obtained from $X$ by killing the $n$-th homotopy group $\pi_{n}(X)$. We obtain inductively $X_{s}^{(t)}$ by the same constiuction, and the sequence of fiber spaces $\left\{X_{s}^{(t)} \rightarrow X_{s-1}^{(t)}\right\}$ is the Postnikov system of $X^{(t)}$ which is obtained from $X^{(t-1)}$ by killing the $(n+t-1)$-th homotopy group of $X^{(t)}$.

Let $\xi_{q}^{t}: X_{q}^{(t)} \rightarrow X_{q}$ be the composition of the maps $\eta_{q}$ 's. Then $\xi_{q}^{t+} k^{q+2}(X)$ is the Postnikov invariant of $X^{(t)}$, and it is a suspension as far as $k^{q+2}(X)$ is. By using the above notations, we obtain the following theorem.

THEOREM 4.3. Let $f: K^{s-1} \rightarrow X$ be extendable over $K^{q^{+1}},(n \leqq s \leqq q \leqq 2 n-3)$. 
Let $f_{0}, f_{1}: K^{s} \rightarrow X$ be two extensions of $f$ which are extendable over $K^{q+1}$. Then $Z_{s}^{q+2}\left(f_{0}\right)$ and $Z_{s}^{q+2}\left(f_{1}\right)$ are cosets of $H^{q+2}\left(K, \pi_{q+1}(X)\right)$ by the subgroup $L^{q+1}\left(\xi_{q}^{s-n *} k^{q+2}(X)\right)$, the difference cocycle $d^{s}\left(f_{0}, f_{1}\right) \in$ Kernel $\left[\xi_{q-1}^{s-n *} k^{q+1}(X)\right]$, and the following relation holds.

$$
Z_{s}^{q+2}\left(f_{0}\right)-Z_{s}^{q+2}\left(f_{1}\right)=\left[\xi_{q}^{s-n *} k^{q+2}(X)\right] d^{s}\left(f_{0}, f_{1}\right) .
$$

\section{Proof of the theorem 4.3}

We divide the theorem into two parts.

$\left(\mathrm{I}_{q, s}\right)$ Let $f: K^{s} \rightarrow X$ be extendable over $K^{q+1}(n \leqq s \leqq q \leqq 2 n-3)$, then $Z_{s}^{q+2}(f)$ is a coset of $H^{q+2}\left(K, \pi_{q+1}(X)\right)$ by the subgroup $L^{q+2}\left(\xi_{q}^{s-n *} k^{q+2}(X)\right)$.

$\left(\mathrm{II}_{q, s}\right)$ Let $f: K^{s-1} \rightarrow X$ be extendable over $K^{q+1}(n \leqq s \leqq q \leqq 2 n-3)$. Let $f_{0}$, $f_{1}: K \rightarrow X$ be two extensions of $f$ which are extendable over $K^{q+1}$. Then $d^{s}\left(f_{0}, f_{1}\right) \in$ Kernel $\left(\left[\xi_{q-1}^{s-n *} k^{q+2}(X)\right]\right)$ and

$$
Z_{s}^{q+2}\left(f_{0}\right)-Z_{s}^{q+2}\left(f_{1}\right)=\left[\xi_{q}^{s-n *} k^{q+2}(X)\right] d^{s}\left(f_{0}, f_{1}\right) .
$$

We shall prove $\left(\mathrm{I}_{q, s}\right)$ and $\left(\mathrm{II}_{q, s}\right)$ by double induction on $(q, s)(n \leqq s \leqq q$ $\leqq 2 n-3)$.

If $s=q, Z_{q}^{q+2}\left(f_{0}\right)$ and $Z_{q}^{q+2}\left(f_{1}\right)$ are single cohomology classes $\bar{c}^{q+2}\left(f_{0}\right)$ and $\bar{c}^{q+2}\left(f_{1}\right)$, and $\xi_{q}^{q-n *} k^{q+2}(X) \in H^{q+2}\left(X_{q}^{(q-n)}, \pi_{q+1}(X)\right) \approx H^{q+2}\left(\pi_{q}(X), q ; \pi_{q+1}(X)\right)$. Therefore, $\left[\xi_{q}^{q-n *} k^{q+2}(X)\right]$ is a usual cohomology operation, and $L^{q+2}\left(\xi_{q}^{q-n *}\right.$ $\left.k^{q+2}(X)\right)$ is the zero group. Therefore, $\left(I_{q, q}\right)$ is proved for any $q$.

We shall prove $\left(\mathrm{I}_{q, s}\right)$ and $\left(\mathrm{II}_{q, s}\right)$ in two steps.

(A) Assuming $\left(\mathrm{I}_{q^{\prime}, s^{\prime}}\right),\left(\mathrm{II}_{q^{\prime}, s^{\prime}}\right)$ for $q^{\prime}<q, n \leqq s^{\prime} \leqq q^{\prime}$ and $\left(\mathrm{I}_{q, s^{\prime}}\right),\left(\mathrm{II}_{q, s^{\prime}+1}\right)$ for $s^{\prime} \geqq s$, we deduce $\left(\mathrm{II}_{q, s}\right)$.

(B) Assuming $\left(\mathrm{I}_{q^{\prime}, s^{\prime}}\right),\left(\mathrm{II}_{q^{\prime}, s^{\prime}}\right)$ for $q^{\prime}<q, n \leqq s^{\prime} \leqq q^{\prime}$ and $\left(\mathrm{I}_{q, s^{\prime}}\right),\left(\mathrm{II}_{q, s^{\prime}}\right)$ for $s^{\prime} \geqq s$, we deduce $\left(\mathrm{I}_{q, s-1}\right)$.

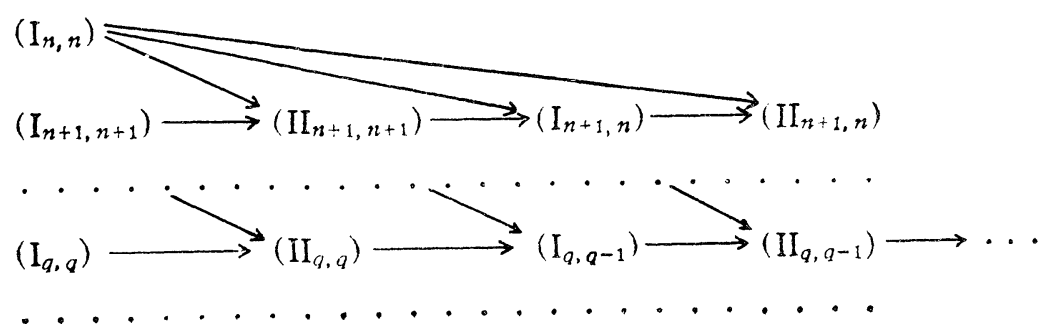


Step (B)

LeMma 5.1. $L^{q+2}\left(\xi_{q}^{s-n-1 \cdot} k^{q: 2}(X)\right)=$ Image $\left[\xi_{q}^{s-n k} k^{q+2}(X)\right]$, where we consider Image $\left[\xi_{q}^{s-n *} k^{q+2}(X)\right]$ as a union of cosets in $H^{q+2}\left(K, \pi_{q+1}(X)\right)$ by $L^{q+2}\left(\xi_{q}^{s-n *} k^{q+2}(X)\right)$.

This follows immediately from the definition.

Step (B) follows from $\left(\mathrm{I}_{q, s}\right),\left(\mathrm{II}_{q, s}\right)$, and Lemma 5.1.

Step (A)

By our assumption $f_{0}, f_{1}$ are extendable over $K^{q+1}$, therefore, $Z_{s}^{q^{\prime}+2}\left(f_{0}\right)$ $=Z_{s}^{q^{\prime}+2}\left(f_{1}\right)=0$ for $q^{\prime}<q$. By $\left(\mathrm{II}_{q^{\prime}, s}\right) \quad\left(q^{\prime}<q\right)$ we have $d^{s}\left(f_{0}, f_{1}\right) \in$ Kernel $\left[\xi_{q-1}^{s-n *} k^{q+1}(X)\right]$. Next, we shall prove the equality.

We can assume that $f\left(K^{n-1}\right)$ is a single point without loss of generality. Let $K_{0}$ be obtained from $K$ by shrinking $K^{n-1}$ to a single point $v_{0}$. Let $h: K$ $\rightarrow K_{0}$ be the shrinking map.

Since $\pi_{i}\left(X_{q}\right)=0$ for $i>q$, and $f_{0}$ is extendabie over $K^{q+1}, p_{q}^{\prime} \circ f_{0}: K^{n} \rightarrow X_{q}$ is extendable over $K$. Let $F: K \rightarrow X_{q}$ be an extension of $p_{q}^{\prime} \circ f_{0}$. Then there exists a map $g: K_{0} \rightarrow X_{q}$ such that $g \circ h=F$.

Take a $s$-cell $\Delta_{i}^{s}$ in the interior of each $s$-cell $\sigma_{l}^{s}$ of $K$ except one common vertex. Let $K_{0} \vee X_{q}^{(s-n)}$ be a union of $K_{0}$ and $X_{q}^{(s-n)}$ with a single point in common. We shall construct a map $k: H^{q+2} \rightarrow K_{0} \vee X_{q}^{(s-n)}$ as follows.

Let $k_{s}: K^{s} \rightarrow K_{0} \vee X_{q}^{(s-n)}$ be a map such that

$$
k_{s}\left|K^{s-1}=i_{1} \circ h\right| K^{s-1} \text {, }
$$

where $i_{1}: K_{0} \rightarrow K_{0} \vee X_{q}^{(s-n)}$ is the inclusion map,

(2) $k_{s}$ maps $\sigma_{i}^{s}-\Delta_{i}^{s}$ homeomorphically onto $\sigma_{i}^{s}-v_{0}$ in $K_{0}$,

(3) $k_{s}$ maps the boundary of $\Delta_{i}^{s}$ to $v_{0}$ in $K_{0}$, and

(4) $k_{s}$ maps $\left(\Delta_{i}^{\varsigma}\right.$, boundary of $\left.\Delta_{i}^{\S}\right)$ into $\left(X_{q}^{(S-n)}, v_{0}\right)$ by a map corresponding to $d^{s}\left(f_{1}, f_{0}\right)\left(\sigma_{i}^{s}\right) \in \pi_{s}(X) \approx \pi_{s}\left(X_{q}^{(s-n)}\right)$.

Then $c^{s+1}\left(k_{s}\right)=\delta d^{s}\left(f_{1}, f_{0}\right)=0$. Therefore, $k_{s}$ is extendable over $K^{s+1}$.

Consider the following diagram.

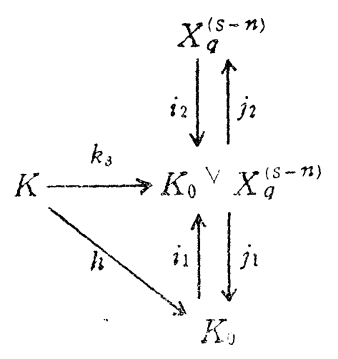


where $j_{1}, j_{2}$ are natural projections, and $i_{1}, i_{2}$ are inclusion maps. Then it is easily seen that

$$
\begin{aligned}
& j_{1} \circ k_{s} \sim h \quad \text { on } K^{s}, \text { and } \\
& d^{s}\left(k_{s}, i_{1} \circ h\right)=d^{s}\left(f_{1}, f_{0}\right) .
\end{aligned}
$$

Suppose $k_{s}$ be extendable ober $K^{r+1}, r<q$. Since

$$
\pi_{i}\left(K_{0} \vee X_{q}^{(s-n)}\right) \approx \pi_{i}\left(K_{0}\right)+\pi_{i}\left(X_{q}^{(s-n)}\right) \quad \text { for } i \leqq n+s-2,
$$

$Z_{s}^{r+2}\left(k_{s}\right)$ is decomposed into two terms, i.e. one in coefficients in $\pi_{r+1}\left(K_{0}\right)$ and the other in coefficients in $\pi_{r+1}\left(X_{q}^{(s-n)}\right)$ for $r<q \leqq 2 n-3$. The first term is zero since $j_{1} \circ k_{s}: K^{s} \rightarrow K_{0}$ is homotopic to $h$, and $h$ is defined over $K$. By $\left(\mathrm{II}_{r, s}\right)(r<q)$ we have

$$
\begin{aligned}
Z_{s}^{r+2}\left(k_{s}\right)-Z_{s}^{r+2}\left(i_{1} \circ h\right) & =\left[\xi_{r}^{s-n *} k^{r+2}(X)\right] d^{s}\left(k_{s}, i_{1} \circ h\right) \\
& =\left[\xi_{r}^{s-n *} k^{r+2}(X)\right] d^{s}\left(f_{0}, f_{1}\right) .
\end{aligned}
$$

But $Z_{s}^{r+2}\left(i_{1} \circ h\right)=0$, and $\left[\xi_{r}^{s-n *} k^{r+2}(X)\right] d^{s}\left(f_{0}, f_{1}\right)=0$ by our assumption. Therefore $Z_{s}^{r+2}\left(k_{s}\right)=0$ for $r<q$, and $k_{s}$ is extendable over $K^{q+1}$.

Since $q+1 \leqq n+s-2, Z_{s}^{q+2}\left(k_{s}\right)=Z_{s}^{q+2}\left(j_{1} \circ k_{s}\right)+Z_{s}^{q+2}\left(j_{2} \circ k_{s}\right) . \quad Z_{s}^{q+2}\left(j_{1} \circ k_{s}\right)$ $=Z_{s}^{q+2}(h)=0$ and $Z_{s}^{q+2}\left(j_{2} \circ k\right)=0$ because $\pi_{q+1}\left(X_{q}^{(s-n)}\right)=0$. Therefore, $Z_{s}^{q+2}\left(k_{s}\right)$ $=0$, and $k_{s}$ is extendable over $K^{q+2}$.

Let $k^{\prime}: K^{q+2} \rightarrow K_{0} \vee X_{q}^{(s-n)}$ be an extension of $k_{s}$. We shall modify $k^{\prime}$ to $k: K^{q+2} \rightarrow K_{0} \vee X_{q}^{(s-n)}$ so that $j_{1} \circ k \sim h$. Let $k_{r+1}^{\prime}: K^{r+1} \rightarrow K_{0} \vee X_{q}^{(s-n)}, r \leqq q$, be extension of $k_{s}$ such that $j_{1} \circ k_{r+1}^{\prime} \sim h$ on $K^{r+1}$, and $j_{2} \circ k_{r+1}^{\prime} \sim j_{2} \circ k^{\prime}$. Then it is clear that $k_{r+1}^{\prime}$ is extendable over $K^{r+2}$. Let $k_{r+2}^{\prime \prime}$ be an extension of $k_{r+1}^{\prime}$ over $K^{r+2}$. There exists a map $k_{r+2}^{\prime}: K^{r+2} \rightarrow K_{0} \vee X_{q}^{(s-n)}$ such that $d^{r+2}\left(k_{r+2}^{\prime}, k_{r+2}^{\prime \prime}\right)$ $=i_{1 *} d^{r+2}\left(j_{1} \circ k^{\prime \prime}, h\right)+i_{2 *} d^{r+2}\left(j_{2} \circ k_{r+2}^{\prime \prime}, j_{2} \circ k^{\prime}\right)$, where $i_{1 *}$ (resp. $\left.i_{2 *}\right)$ is induced by the coefficients homomorphism $i_{1 *}: \pi_{r+2}\left(K_{0}\right) \rightarrow \pi_{r+2}\left(K_{0} \vee X_{q}^{s-n)}\right.$ ) (resp. $i_{2 *}$ : $\pi r\left\llcorner 2\left(X_{q}^{(s-n)}\right) \rightarrow \pi_{r+2}\left(K_{0} \vee X_{q}^{(s-n)}\right)\right)$. Then it is clear by the simple calculation of the difference cochain that $j_{1} \circ \bar{k}_{r+2}^{\prime} \sim h$ and $j_{2} \circ k_{r+2}^{\prime} \sim j_{2} \circ k^{\prime}$ on $K^{r+2}$. Therefore, finally we have the desired map $k: K^{q+2} \rightarrow K_{0} \vee X_{\eta}^{(s-n)}$ such that $j_{1} \circ k \sim h$.

Then we have the following properties.

$$
\begin{aligned}
& j_{1} \circ k \sim h \\
& \left(j_{2} \circ k\right)^{*} b^{s}\left(X_{q}^{(s-n)}\right)=i^{*} d^{s}\left(f_{1}, f_{0}\right),
\end{aligned}
$$

where $b^{s}\left(X_{q}^{(s-n)}\right) \in H^{s}\left(X_{q}^{(s-n)}, \pi_{s}(X)\right)$ is the fundamental cohomology class and 
$i^{*}: H^{s}(K) \rightarrow H^{s}\left(K^{q+2}\right)$ is the induced homomorphism by the inclusion $i: K^{q+2}$ $\rightarrow K$.

(5.2) follows from the fact that $k$ is an extension of $k_{s}$.

Consider the following diagram.

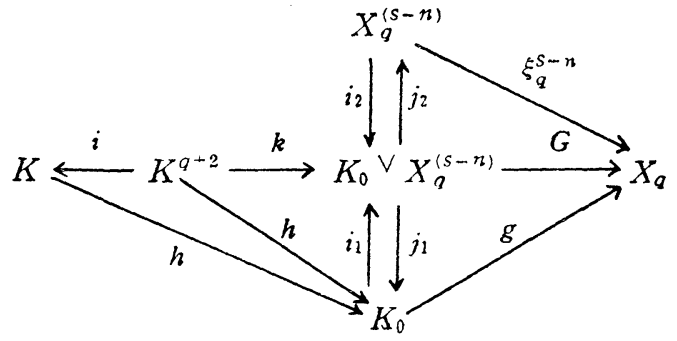

where $G \mid K_{0}=g$ and $G \mid X_{q}^{(S-n)}=\xi_{q}^{s-n}$.

Then we have the following properties.

$$
\begin{aligned}
& G \circ i_{1} \circ h=F \circ i . \\
& G \circ i_{2}=\xi_{q}^{s-n} . \\
& \left(i_{1} \circ h\right)^{*}-k^{*}=-\left(i_{2} \circ j_{2} \circ k\right)^{*}
\end{aligned}
$$

(5.5) follows from (5.1).

By the same argument used in the beginning of this section $p_{q}^{\prime} \circ f_{1}: K^{s} \rightarrow X_{q}$ is extendable over $K$. Let $F^{\prime}$ be an extension of $p_{q}^{\prime} \circ f_{1}$. Then

Therefore,

$$
\begin{aligned}
d^{s}\left(G \circ k, F^{\prime}\right) & =d^{s}(G \circ k, F)+d^{s}\left(F, F^{\prime}\right) \\
& =G_{*} d^{s}\left(k, i_{1} \circ h\right)+d^{s}\left(f_{0}, f_{1}\right) \\
& =d^{s}\left(f_{1}, f_{0}\right)+d^{s}\left(f_{0}, f_{1}\right)=0 .
\end{aligned}
$$

$$
G \circ k \sim F^{\prime} \quad \text { on } K^{s} \text {. }
$$

By Lemma 2.6,

$$
\begin{aligned}
& \bar{c}^{q+2}\left(f_{0}\right)=F^{*} k^{q+2}(X), \text { and } \\
& \bar{c}^{q+2}\left(f_{1}\right)=F^{* *} k^{q+2}(X) .
\end{aligned}
$$

By (5.6) we have $i^{*} \bar{c}^{q+2}\left(f_{1}\right)$ and $(G \circ k)^{*} k^{q+2}(X)$ belong to the same obstruction set $Z_{s}^{q+2}\left(f_{1} \mid K^{q+2}\right)=i^{*} Z_{s}^{q+2}\left(f_{1}\right)$. Therefore, by (5.3), (5.5), and (5.4)

$$
\begin{aligned}
i^{*} & \left(Z_{s}^{q+2}\left(f_{0}\right)-Z_{s}^{q+2}\left(f_{1}\right)\right) \ni(F \circ i)^{*} k^{q+2}(X)-(G \circ k)^{*} k^{q+2}(X) \\
& =\left(\left(i_{1} \circ h\right)^{*}-k^{*}\right) G^{*} k^{q+2}(X)=-\left(i_{2} \circ j_{2} \circ k\right)^{*} G^{*} k^{q+2}(X) \\
& =-\left(j_{2} \circ k\right)^{*} i_{2}^{*} G^{*} k^{q^{+2}}(X)=-\left(j_{2} \circ k\right)^{* *} \xi^{s-n *} k^{q+2}(X) .
\end{aligned}
$$


On the other hand, $\xi_{q}^{s-n *} k^{q+2}(X) \in\left[\xi_{q}^{s-n *} k^{q+2}(X)\right] b^{s}\left(X_{q}^{(s-n)}\right)$ by definition. Therefore, by (5.2)

$$
\begin{aligned}
& i^{*}\left(Z_{s}^{q+2}\left(f_{0}\right)-Z_{s}^{q+2}\left(f_{1}\right)\right)=-\left(j_{2} \circ k\right)^{*}\left[\xi_{q}^{s-n *} k^{q+2}(X)\right] b^{s}\left(X_{q}^{(s-n)}\right) \\
& \quad=-\left[\xi_{q}^{s-n *} k^{q+2}(X)\right]\left(j_{2} \circ k\right)^{*} b^{s}\left(X_{q}^{(s-n)}\right) \\
& \quad=-\left[\xi_{q}^{s-n *} k^{q+2}(X)\right] i^{*} d^{s}\left(f_{1}, f_{0}\right) \\
& \quad=i^{*}\left[\xi_{q}^{s-n *} k^{q+2}(X)\right] d^{s}\left(f_{0}, f_{1}\right) .
\end{aligned}
$$

Since $i^{*}$ is an isomorphism into, we have the desired equality, i.e.

$$
Z_{s}^{q+2}\left(f_{0}\right)-Z_{s}^{q+2}\left(f_{1}\right)=\left[\xi_{q}^{s-n *} k^{q+2}(X)\right] d^{s}\left(f_{0}, f_{1}\right) .
$$

\section{Supplementary result}

Theorem 6.1. Let $X$ be 2-connected $C W$-complex. Let $f: K^{q-1} \rightarrow X$ be a map $(q \geqq 2)$ which is extendable over $K^{p+1}$. Let $f_{0}$ and $f_{1}$ be two extensions of $f$ over $K^{q}$ which are extendable over $K^{q+1}$. Then

$$
\bar{c}^{q+2}\left(f_{0}\right)-\bar{\sigma}^{q+2}\left(f_{1}\right)=S q^{2} d^{q}\left(f_{0}, f_{1}\right),
$$

where $S q^{2}: H^{q}\left(K, \pi_{q}(X)\right) \rightarrow H^{q^{+2}}\left(K, \pi_{q+1}(X)\right)$ is the squaring operation.

Proof. With a little modification of the proof of Theorem 4.3, we can prove

$$
\bar{c}^{q+2}\left(f_{0}\right)-\bar{c}^{q+2}\left(f_{1}\right)=\left[\xi_{q}^{q-3 *} k^{q+2}(X)\right] d^{q}\left(f_{0}, f_{1}\right) .
$$

$\xi_{q}^{q-3 *} k^{q+2}(X)$ belongs to $H^{q+2}\left(X_{q}^{(q-3)}, \pi_{q+1}(X)\right)$ and $X$ is 2-connected. Therefore, $X_{q}^{(q-3)}$ has only one non vanishing homotopy group $\pi_{q}(X)$ in dimension $q$, and $\xi_{q}^{q-3 *} k^{q+2}(X)$ is the Eilenberg-MacLane invariant of the space $X_{q+1}^{(q-3)}$, which has two non-vanishing homotopy groups in dimensions $q$ and $q+1$. Therefore, it is well-known that

$$
\xi_{q}^{q-3 *} k^{q+2}(X)=S q^{2} b^{q}\left(X_{q+1}^{(q-3)}\right),
$$

i.e. $\left[\xi_{q}^{q-3 *} k^{q+2}(X)\right]=S q^{2}$.

\section{BIBLIOGRAPHY}

[1] S. Eilenberg, Cohomology and continuous mappings, Ann. of Math., 41 (1940), 231-251.

[2] J. C. Moore, Semi-simplicial complexes and Postnikov systems, mimeographed note, Princeton University, (1957).

[3] P. Olum, Obstructions to extensions and homotopies, Ann. of Math., 52 (1950), 1-50.

[4] F. P. Peterson, Functional cohomology operations, Trans. Amer. Math. Soc., 86 (1957), 197-211. 
[5] M. M. Postnikov, Investigations in homotopy theory of continuous mappings, Trudy Mat. Inst. Steklov, no. 46. Izdat. Akad. Nauk SSSR, Moscow, 1955 (in Russian).

[6] N. Shimada, Homotopy classification of mappings of a 4-dimensional complex into a 2-dimensional sphere, Nagoya Math. Jour., 5 (1953), 127-144.

[7] N. E. Steenrod, Products of cocycles and extensions of mappings, Ann. of Math., 48 (1947), 290-320.

[8] J. H. C. Whitehead, On the theory of obstructions, Ann. of Math., 54 (1951), 68-84.

Mathematical Institute

Nagoya University 